\title{
Rescattering of Ultra Low-Energy Electrons for Single Ionization of Ne in the Tunneling Regime
}

\author{
R. Moshammer ${ }^{*}$, J. Ullrich ${ }^{1}$, B. Feuerstein ${ }^{1}$, D. Fischer ${ }^{1}$, A. Dorn ${ }^{1}$, C.D. Schröter ${ }^{1}$, J.R. \\ Crespo Lopez-Urrutia ${ }^{1}$, C. Hoehr ${ }^{1}$ \\ ${ }^{1}$ Max-Planck-Institut für Kernphysik, Saupfercheckweg 1, D-67119 Heidelberg, Germany
}

\author{
H. Rottke ${ }^{2}$, C. Trump ${ }^{2}$, M. Wittmann ${ }^{2}$, G. Korn², W. Sandner ${ }^{2}$ \\ ${ }^{2}$ Max-Born-Institut, Max-Born-Str 2a, D-12489 Berlin, Germany \\ *e-mail: R.Moshammer@mpi-hd.mpg.de
}

\begin{abstract}
Electron emission for single ionization of $\mathrm{Ne}$ by $25 \mathrm{fs}, 1.0 \mathrm{PW} / \mathrm{cm}^{2}$ laser pulses at $800 \mathrm{~nm}$ has been investigated in a kinematically complete experiment using a "reaction-microscope". Mapping the complete final state momentum space with high resolution a distinct minimum along the longitudinal momentum direction $\mathrm{P}_{\mathrm{e} \|}$ is observed at $\mathrm{P}_{\mathrm{e} \|}=0$ with respect to the laser polarization direction. Whereas tunneling theory predicts a maximum at the singularity our findings are in good agreement with recent semiclassical predictions which were interpreted to be due to "recollision".
\end{abstract}

PACS numbers: 32.80.Rm, 32.80.Fb, 32.90.+a, 42.50.Hz 
A profound knowledge of the electron dynamics in intense, femtosecond laser fields interacting with single atoms or molecules is indispensable for our understanding of laser-matter interactions in general and, thus, for a large body of present and future applications, like high harmonic generation, acceleration of electrons and ions, strong field induced fusion or the realization of intense laboratory $\mathrm{x}$-ray sources. Fueled by results of novel many-particle imaging techniques $[1,4]$ recent interest in intense laser-atom interaction has increasingly focused to the investigation of many-electron transitions like double and multiple ionization, experimentally [2-9] as well as theoretically (see e.g. [9-16]).

Surprisingly, however, at $\mathrm{PW} / \mathrm{cm}^{2}$ intensities, where recollision is now being generally accepted [2-16] to dominate non-sequential double ionization, only few differential electron emission studies can be found in the literature for single ionization by femtosecond pulses in the optical frequency regime [17-22]. Here, at typical Keldysh [23] parameters $\gamma=\left(I_{P} / 2 U_{P}\right)^{1 / 2} \ll 1\left(I_{P}\right.$ : ionization potential; $\mathrm{U}_{\mathrm{P}}=\mathrm{I} /(2 \omega)^{2}$ : ponderomotive potential with $\mathrm{I}$ the laser intensity and $\omega$ its frequency; atomic units are used throughout), the photoelectron spectra become increasingly smooth and unstructured, whereas they display a confusingly rich structure at lower intensities for $\gamma \geq 1$.

Theoretically, the smooth exponential-like decrease has been explained early by quasi-static tunneling $[17,23,24]$ of the active electron into the continuum or by treating above threshold ionization quantum mechanically as a transition of the bound electron to free electron states oscillating in the electromagnetic field (Volkov states) of the laser (the so-called Keldish- 
Faisal-Reiss, KFR-model $[23,25,26])$. In tunneling theory the active electron tunnels in a first step and is then accelerated, gaining drift energy in the oscillating field in a second step. The drift energy is a smooth function of the tunneling phase in the field with zero energy for tunneling at the maximum of the field and a maximum energy of up to $2 U_{P}$ for tunneling at a phase where the field is zero. Immediately, interest concentrated nearly exclusively on large electron energies, beyond $2 U_{P}$, where significant deviations from simple tunneling as well as KFR-models were recognized and explained later by rescattering of the oscillating electron on its parent ion (see e.g. $[21,22,24])$ causing acceleration up to a maximum energy of $10 U_{P}$.

Until now, the very low-energy part of the electron spectrum at large laser intensities, close or even at zero electron energy, has never really been considered experimentally as well as theoretically. Since tunneling or over the barrier ionization is most likely at phases of the optical field where the electric field maximizes, essentially all theoretical approaches predict a maximum at zero electron energy in accord with most of the few existing experimental data that reliably extend to very low energies. Unexplained structures at low energies, (a peak at $10 \mathrm{eV}$ and a "cut-off" at about $2.5 \mathrm{eV}$, slightly above the lowest value of about $2 \mathrm{eV}$ from where on the electron spectra were presented) were only reported to the best of our knowledge by Mohideen et al. [18] "in disagreement" with all theoretical predictions and with results of other measurements [19-22]

Essentially "en passant" very recent experiments [3,4,7-9], originally devoted to measure electron spectra for double ionization, reported on low- 
energy single ionization electron spectra $\left(E_{e}<150 \mathrm{eV}\right)$. All of them at least find increasing yields for decreasing electron energies or, some of them, even a maximum at zero energy $[3,4,7,8]$, as expected. Most notably, a very clear maximum at zero momentum was observed in both recoil-ion momentum measurements [3,4]. Ion momenta close or even at zero can be reliably determined as proven in many previous investigations and are absolutely equivalent to the electron momenta [6]. Thus, indirectly, differential electron spectra have been recorded down to zero emission energy within the momentum resolution achieved, which was 0.2 a.u. for the $\mathrm{Ne}$ measurement and was not stated [3] but probably worse for the helium experiment [27].

In this Letter we report on first high-resolution, fully differential ultra-low energy electron emission spectra for single ionization of $\mathrm{Ne}$ by 25 fs 1.0 $\mathrm{PW} / \mathrm{cm}^{2}$ laser pulses under unprecedented clean experimental conditions. Well in the tunneling regime $(\gamma=0.34)$ we find a distinct minimum at zero electron energy in striking contradiction to essentially all standard theoretical predictions and most of the above-mentioned experiments. Using our "reaction microscope" the complete final state momentum space, i.e. electron as well as ion energy and angular distributions, has been recorded for all electrons with energies $\mathrm{E}_{\mathrm{e}}<30 \mathrm{eV}$ with an excellent $\left(\Delta \mathrm{P}_{\perp, \|}<0.1\right.$ a.u. $)$, well-defined and controlled momentum resolution.

The coincident detection of the recoiling $\mathrm{Ne}^{1+}$ ion momentum vector first ensures that no contributions from higher charge states obscure the electron spectra. Second, and even more important, space-charge effects that would influence the final momentum distributions of electrons and ions very 
differently can be safely excluded since we observe momentum conservation between the electron and the $\mathrm{Ne}^{+}$ion $\mathbf{P}_{\mathrm{e}}=-\mathbf{P}_{\mathrm{Ne}}$ for each single event (neglecting the small photon momentum transfer). Third, identical momenta within the experimental resolution further guarantee that the electron spectra are not noticeably influenced by any ponderomotive acceleration in the inhomogeneous field of the laser focus since this again would act very differently on electrons and ions for essentially the same reason: Whereas the ions basically stick at the same location within the laser pulse due to their low velocity $\left(\mathrm{v}_{\mathrm{Ne}} \leq 120 \mathrm{~m} / \mathrm{s}=3 \cdot 10^{-12} \mathrm{~m}\right.$ in $\left.25 \mathrm{fs}\right)$ electrons with identical momenta of up to 2 a.u. are considerably faster as a result of their low mass and, thus, in principle might be affected.

The experiments were performed at the Max-Born Institute in Berlin using a Kerr-lens mode locked Ti:Sapphire laser at $800 \mathrm{~nm}$ wavelength with a $1 \mathrm{kHz}$ repetition rate. The pulses of 25 fs length (FWHM) were focussed ( $f=100$ $\mathrm{mm}$ ) to a spot of $10 \mu \mathrm{m}$ diameter (FWHM) into an ultrahigh vacuum chamber $\left(2 \cdot 10^{-11}\right.$ Torr $)$. The light intensity of $1.0 \mathrm{PW} / \mathrm{cm}^{2}$ has been determined from the optically measured focal spot size and the pulse energy. At its focus the laser beam was crossed by a low-density $\left(10^{8}\right.$ atoms $\left./ \mathrm{cm}^{3}\right)$ supersonic gas jet formed by expanding $\mathrm{Ne}$ atoms at a pressure of 5 bar through a $\mathrm{LN}_{2}$ cooled, $10 \mu \mathrm{m}$ diameter nozzle. The atomic beam was collimated over a total length of $2 \mathrm{~m}$ resulting in an interaction volume of $50 \mu \mathrm{m}$ length and $10 \mu \mathrm{m}$ diameter. Using a reaction-microscope, which has been described in detail $[1,2]$, the ionic charge as well as the momentum vectors of both, ions and electrons, were 
recorded in coincidence with high resolution. Intensity fluctuations of the laser were monitored during the experiment and kept below $5 \%$.

All momentum distributions presented in this paper have been projected onto the plane perpendicular to the jet expansion where the ion momentum resolution is best $\left(\Delta \mathrm{P}_{\mathrm{R}} \approx 0.1\right.$ a.u. $)$. Due to the axial symmetry along the laser polarization direction these spectra contain the full information. The ion momentum resolution has been experimentally confirmed by inspecting the sum momentum of electrons and ions for each single event in both directions and a FWHM of the $\left(\mathrm{P}_{\mathrm{e} \|}^{2}+\mathrm{P}_{\mathrm{R} \|}^{2}\right)^{1 / 2}$ distributions of \pm 0.055 a.u. has been achieved [6]. Even if final electron energies of up to about $30 \mathrm{eV}$ where observed requiring the effective absorption of about 30 photons from the radiation field their total sum momentum amounts only to about 0.01 a.u. and, thus, can be safely neglected in the total balance.

In Fig. 1 two-dimensional electron and ion momentum distributions are shown for single ionization of $\mathrm{Ne}$. Whereas for the ions the complete distribution has been recorded simultaneously, the electron spectrum is directly measured only for all $\mathrm{P}_{\mathrm{e} \|}<-0.7$ a.u.. Electrons with $\mathrm{E}_{\mathrm{e} \|}>6.6 \mathrm{eV}$ into the direction opposite to the electron detector leave the spectrometer are not registered. Since the spectrum has to be mirror symmetric with respect to $\mathrm{P}_{\mathrm{e} \|}=$ 0, a complete spectrum has been generated by randomly assigning “+” or "_." signs to all electrons with $\mathrm{P}_{\mathrm{e} \|}>0$.

A widely smooth distribution in the longitudinal as well as transverse direction is observed as expected at high intensities for $\gamma=0.34$. Surprisingly, and in striking contrast to the predictions of tunneling theory, however, a 
minimum is found at $\mathrm{P}_{\mathrm{e} \|}=0$. Since ions and electrons are measured independently, the existence of the minimum is clearly demonstrated. Its form is slightly different in both distributions and less pronounced in the ion spectrum due to the somewhat worse momentum resolution for $\mathrm{Ne}^{1+}$ as compared to the electrons. In a first measurement [4], where only ions were recorded the momentum resolution was limited to $\Delta \mathrm{P}=0.2$ a.u. and, hence, the sharp minimum was not visible at all. As mentioned before, structures at low energies and maybe a minimum at $\mathrm{P}_{\mathrm{e} \|}=0$ have only once been observed in the tunneling regime to the best of our knowledge by Mohideen et al. [18] under much less controlled experimental conditions and, accordingly, have not been discussed since then.

In order to further elucidate our findings we have projected the twodimensional electron distribution onto the longitudinal axis for all transverse momenta in Figure 2. Also plotted is the tunneling theory prediction for 0.7 $\mathrm{PW} / \mathrm{cm}^{2}$ from Delone and Krainov [28] (thin line) with a maximum at $\mathrm{P}_{\mathrm{e} \|}=0$. Here, the electrons have a phase dependent energy directly after tunneling. Then, they are propagated classically in the oscillating electric field $\vec{E}(t)=\vec{E}_{0}(t) \cdot \sin (\omega \cdot t)$ gaining an additional phase dependent $\left(\Phi=\omega \cdot t_{0}\right)$ drift momentum along the polarization direction $P_{d r i f t}\left(t_{0}\right)=q \cdot \omega^{-1} \cdot E_{0}\left(t_{0}\right) \cdot \cos \left(\omega \cdot t_{0}\right)$. Since tunneling is most likely at the maximum of the field $(\Phi=\pi / 2)$ and since those electrons receive zero drift momentum a maximum of the electron momentum distribution at $\mathrm{P}_{\mathrm{e} \|}=0$ is predicted. Furthermore, a Gaussian shape is obtained without further structure which is not in agreement with the present results. 
Also shown in Figure 2 is the result of a recent semiclassical calculation (thick line) for an intensity of $0.7 \mathrm{PW} / \mathrm{cm}^{2}$ and a helium target [29]. It clearly shows not only a minimum at $\mathrm{P}_{\mathrm{e} \|}=0$ but also deviations from the Gaussian shape. Here, electrons are set into the continuum with a velocity distribution obtained from the wave function of the tunneled first electron to model single ionization. Then, the set of trajectories is propagated according to Newton's classical equations in the combined field of the laser and of the Coulomb potential of the residual singly charged helium ion. The ion core is also modeled classically assuming the electron to be in the $\mathrm{He}^{+}$ground state represented by a microcanonical distribution. In this way all mutual interactions between all particles are accounted for during the whole laser pulse in three dimensions. Within this model both, the minimum at zero momentum as well as the deviations from the Gaussian-shape are a result of the interaction of the tunneled electron with its parent ion, i.e. a consequence of rescattering. Switching off the interaction with the parent ion causes both characteristic features to disappear and the tunneling-theory result is recovered [29].

The following interpretation is presented in Ref. [29]. Electrons tunneling close to the maximum of the field experience multiple recurrences to the parent ion with low velocities and, accordingly, large elastic scattering cross sections. Thus, after acceleration in the laser field they are effectively redistributed to larger momenta depleting at the same time the intensity at $\mathrm{P}_{\mathrm{e}}$ close to zero. Since recent purely classical "over-the-barrier" calculations on strong field single ionization [30] did not find a minimum at zero momentum, 
the present findings seem to be closely connected with the quantum nature of the first "tunneling" step. The width of the experimentally observed momentum distribution is considerably smaller than predicted by both, the semiclassical and the tunneling theory. This feature, which has also been found in our previous measurement [4], might either be due to an incorrect determination of the experimental intensity within the estimated accuracy of $20 \%$ or be a result of the intensity integration over the waist of the laser focus in the experiment.

The experimentally observed minimum remains visible even if the doubly differential spectra are transformed into a singly differential distribution as a function of the electron energy, integrated over all emission angles as shown in Figure 3. At the present intensity we otherwise observe a smooth, strongly decreasing energy spectrum without any indication of ATI peaks so that one might safely conclude that the only maximum obtained at about $1 \mathrm{eV}$ is not a remnant of any multi-photon structure.

In summary, we have mapped the final state momentum space of lowenergy electrons with high resolution for single ionization of $\mathrm{Ne}$ by 1.0 $\mathrm{PW} / \mathrm{cm}^{2}, 25 \mathrm{fs}$ laser pulses. A minimum at zero longitudinal momentum is observed on an otherwise smooth distribution which, however, deviates from a Gaussian shape. Both features are in contradiction with simple tunneling theory. On the other hand our data are in convincing agreement with results of a recent semiclassical calculation which is based on tunneling theory but takes the interaction of the oscillating electron with its parent nucleus into account. Whereas effects from recollision have been clearly observed and are 
commonly accepted to be of decisive importance at high electron energies beyond $2 U_{P}$, effects due to recollision have not been observed for extremely small electron momenta at essentially zero electron energy to the best of our knowledge.

From the comparison with theory we are thus lead to the conclusion, that rescattering produces a double-hump structure in the longitudinal momentum distributions of the electron and of the ion, not only for double but, surprisingly, also for single ionization by strong laser fields.

Acknowledgement: This work was supported by the Deutsche Forschungsgemeinschaft (DFG, RO1929) and by the Leibniz-Programm of the DFG. 


\section{Figure Captions:}

Fig.1: Longitudinal and transverse momentum distribution of $\mathrm{Ne}^{1+}$ recoil ions (a) and of electrons (b) for single ionization by $1.0 \mathrm{PW} / \mathrm{cm}^{2}, 25 \mathrm{fs}$ laser pulses at $800 \mathrm{~nm}$. The $\mathrm{z}$-scale is linear with the box sizes proportional to the number of events at a given momentum.

Fig.2: Longitudinal momentum distribution of the emitted electron $\left(\mathrm{P}_{\mathrm{e} \|}\right)$ for the same conditions as in Figure 1. Thin line: prediction of tunneling theory [28] for $0.7 \mathrm{PW} / \mathrm{cm}^{2}$. Thick line: Prediction of semiclassical calculation for $0.7 \mathrm{PW} / \mathrm{cm}^{2}$ single ionization of helium [29].

Fig.3: Electron energy distribution (integrated over all emission angles) for the same conditions as in Figures 1 and 2. 


\section{References:}

[1] J. Ullrich et al., J. Phys. B. 30, 2917 (1997), Topical Review

[2] R. Dörner et al., Advances in At. Mol. Opt. Phys, 48, 1 (2002)

[3] T. Weber et al., Phys. Rev. Lett. 84, 443 (2000);

[4] R. Moshammer et al., Phys. Rev. Lett. 84, 447 (2000)

[5] Th. Weber et al., Optics Express 8, 368, (2001)

[6] R. Moshammer et al., Optics Express 8, 368, (2001)

[7] E.R. Peterson and P.H. Bucksbaum, Phys. Rev. A 64, 053405 (2001)

[8] R. Lafon et al., Phys. Rev. Lett. 86, 2762 (2001)

[9] B. Witzel, N.A. Papadogiannis, and D. Charalambidis, Phys. Rev. Lett. 85, 2268 (2000)

[10] A. Becker and F.H.M. Faisal, Phys. Rev. Lett. 84, 3546 (2000)

[11] M. Lein, E.K.U. Gross, and V. Engel, Phys. Rev. Lett. 85, 4707 (2000)

[12] S.L. Haan, N. Hoekema, S. Poniatowski, Optics Express 7, 29 (2000)

[13] S. P. Goreslavskii et al., Phys. Rev. A 64 (2001) 053402

[14] J. S. Parker, et al., J. Phys. B 33, L691 (2001); 34, L69 (2001)

[15] R. Kopold et al., Phys. Rev. Lett. 85, 3781 (2000)

[16] Li-Bin Fu, Jie Liu, and Shi-Gang Chen Phys. Rev. A 65, 021406 (2002)

[17] B.P. Corkum, N.H. Burnett, and F. Brunel Phys. Rev. Lett. 621259 (1989)

[18] Mohideen et al., Phys. Rev. Lett. 71, 509 (1993)

[19] E. Mevel et al., Phys. Rev. Lett. 70, 406 (1993)

[20] B. Walker et al., Phys. Rev. Lett. 73, 1127 (1994)

[21] B. Walker et al., Phys. Rev. Lett. 77, 5031 (1996) 
[22] B. Sheehy et al., Phys. Rev. A 58, 3942 (1998)

[23] L.V. Keldysh, Zh. Eksp. Teor. Fiz. 47, 1945 (1964)

[24] N.B. Delone, and V.P. Krainov, Physics - Uspekhi 41 (5) 469 (1998)

[25] F.H.M. Faisal J. Phys. B 6, L89 (1973)

[26] H.R. Reiss Phys. Rev. A 22, 1786 (1980); J. Phys. B 20, L79 (1987)

[27] R. Dörner, private communication

[28] N.B. Delone, and V.P. Krainov, J. Opt. Soc. Am. B 8, 1207 (1991).

[29] J. Chen and C.H. Nam, Phys. Rev. A 66, 053415 (2002)

[30] C.R. Feeler and R.E. Olson J. Phys. B 33 (1997 (2000) 


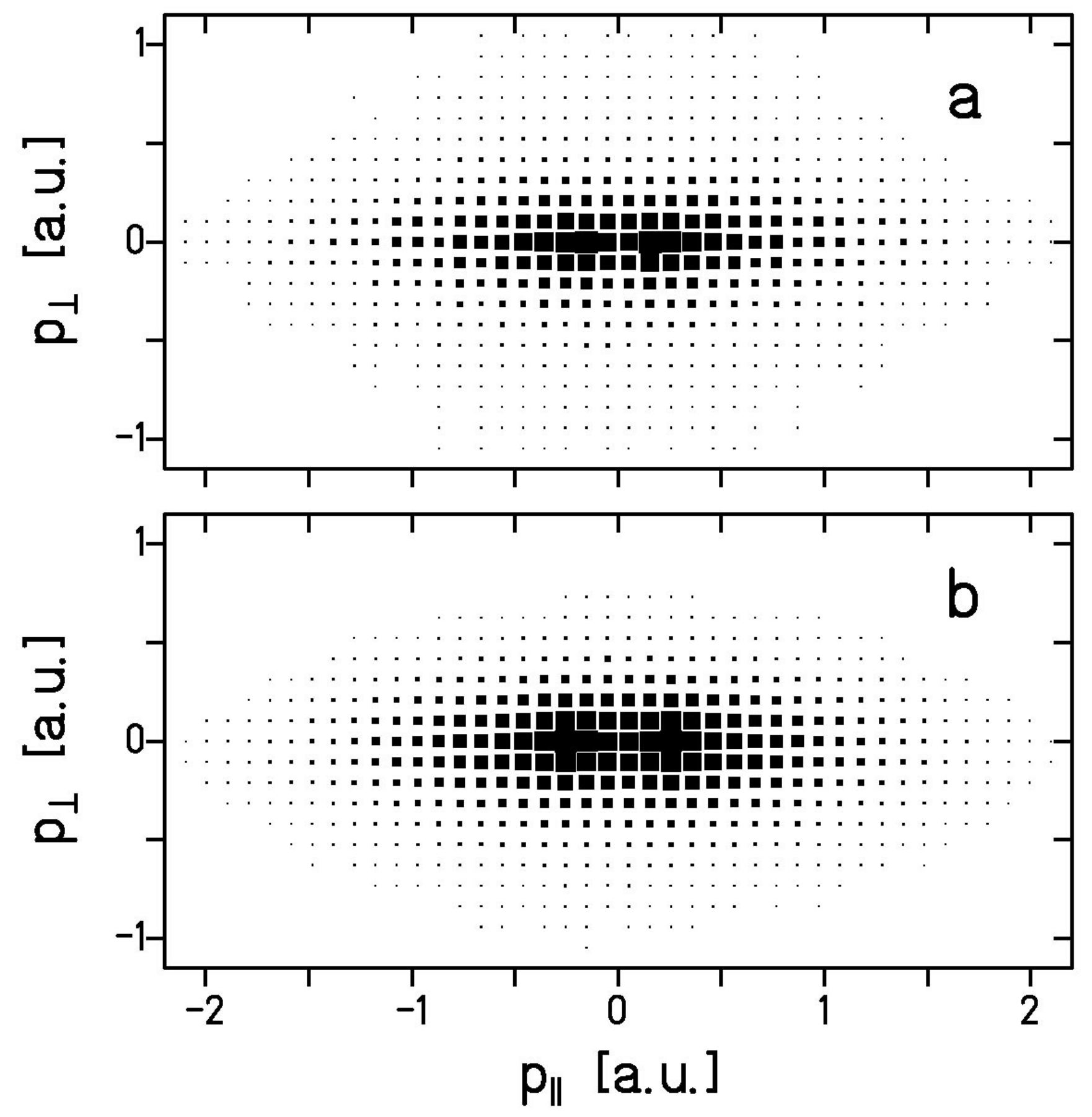

Figure 1 


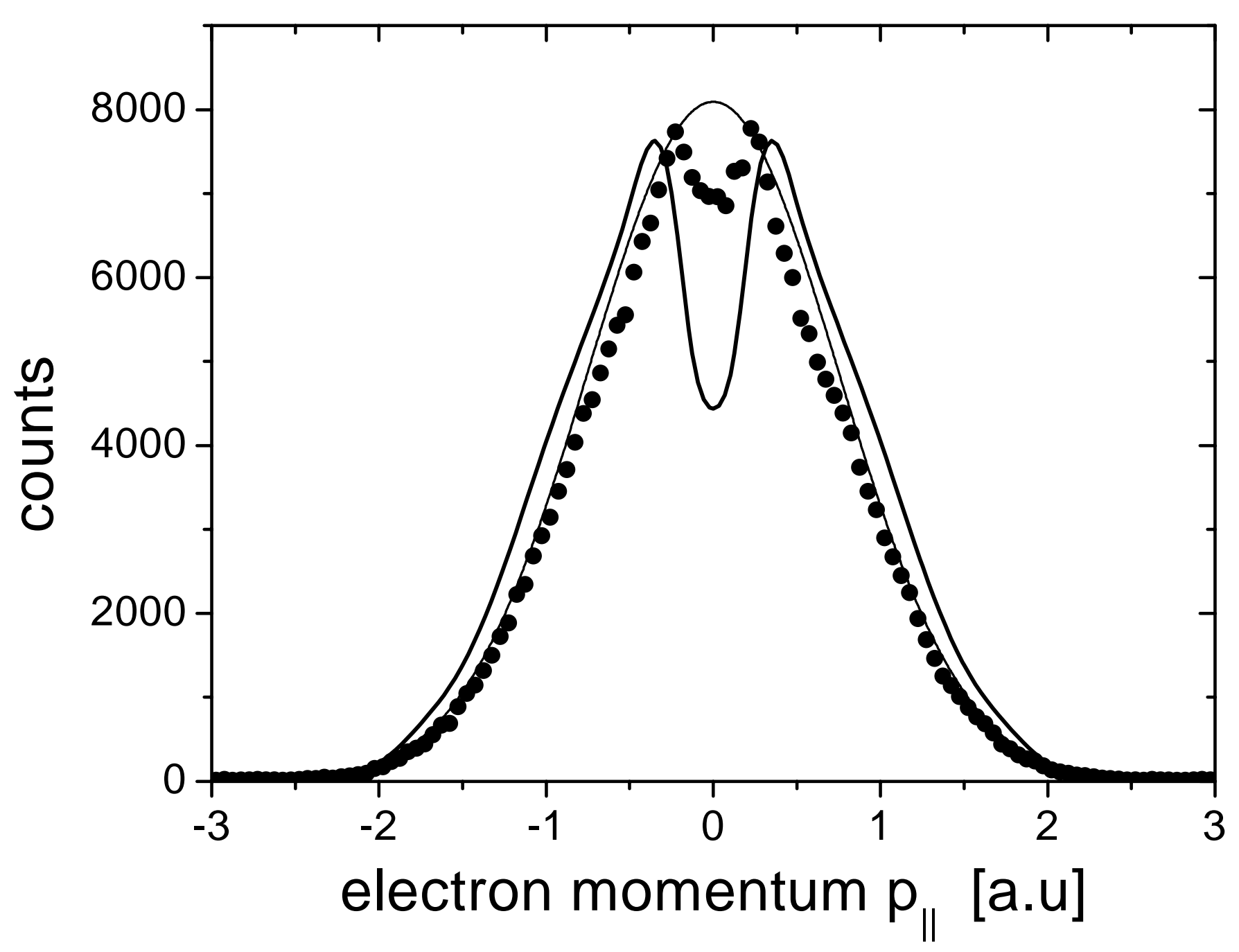

Figure 2 


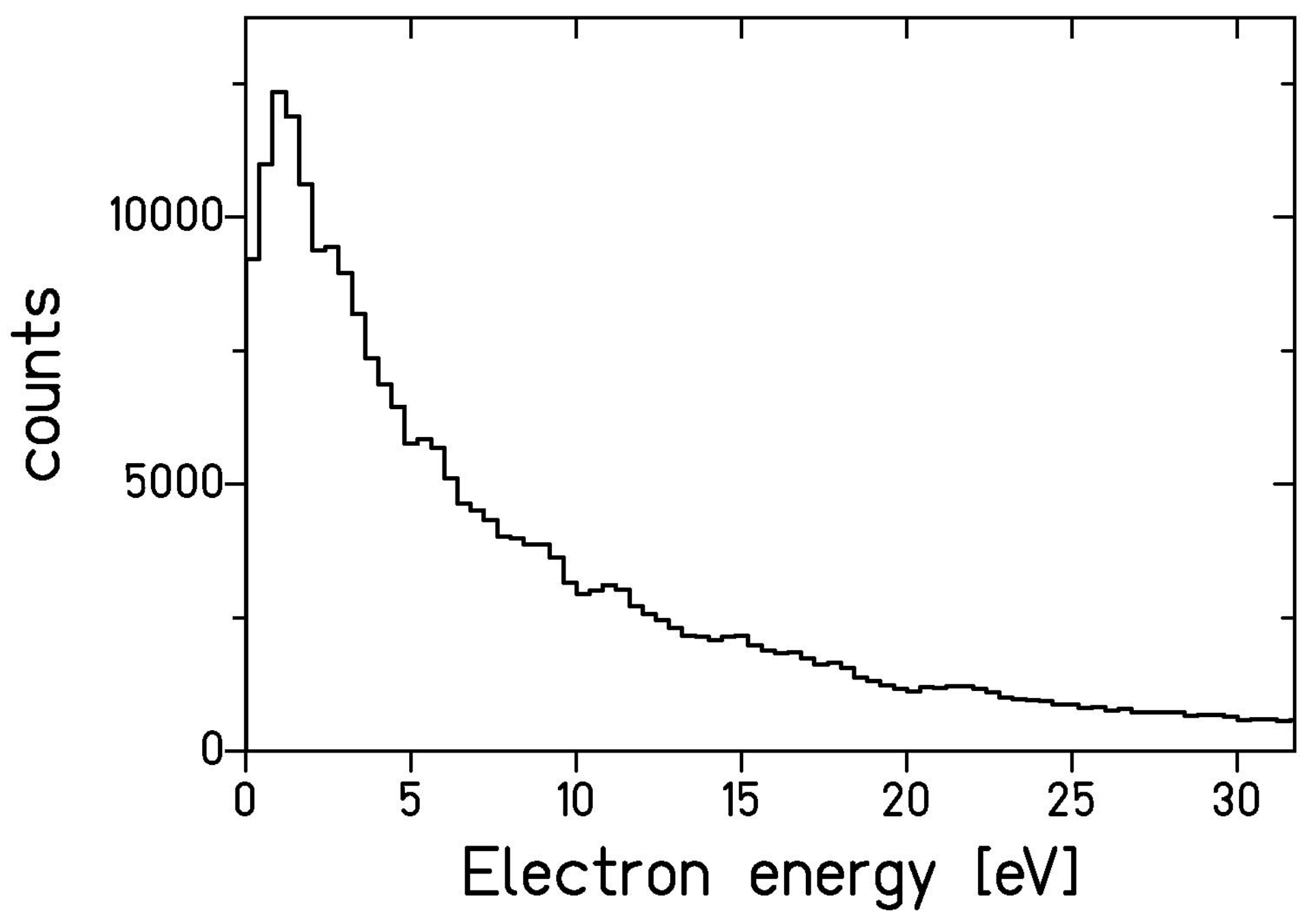

Figure 3 